\section{PROBABILISTIC ANALYSIS OF} THE MAXIMAL LOAD OF INDUSTRIAL MACHINES

ZDENEK FOLTA, PAVEL SKALNY, PETR MATEJKA, MIROSLAV TROCHTA, DANIEL PISTACEK

Department of Machine Parts and Mechanisms,

VSB-Technical University of Ostrava, Ostrava, Czech Republic

Department of Mathematics and Descriptive Geometry

VSB-Technical University of Ostrava, Ostrava, Czech Republic Alliance Laundry CE s.r.o., Pribor, Czech Republic Department of Machine Parts and Mechanisms,

VSB-Technical University of Ostrava, Ostrava, Czech Republic Department of Machine Parts and Mechanisms,

VSB-Technical University of Ostrava, Ostrava, Czech Republic

DOI: 10.17973/MMSJ.2021_12_2021106

zdenek.folta@vsb.cz

This study describes the statistical analysis of peak forces of industrial washing machines. The data source comes from twelve different machines. The measurements are done using a force gauge installed in places for fastening screws. A new software based on LabView has been developed to gauge the acting forces. To determine extreme force values, various probability distributions are applied. Furthermore, a convex combination of lognormal distribution is used in more complicated cases. The parameters of the lognormal mixtures are determined using modified an Expectation-maximization algorithm. Finally, the achieved results are interpreted with regard to the engineering design and to the operating reliability.

KEYWORDS

Washing machine, measurements, force, distribution, probability.

\section{INTRODUCTION}

During the operation process of industrial machines, the components of the machine are loaded with several operational forces. In the case of an industrial washing machine the operational load on the ground must be considered during the installation of the machine. The load is a combination of a static weight and dynamic forces. The dynamic load is mainly caused by the positioning of the laundry and its disbalance.

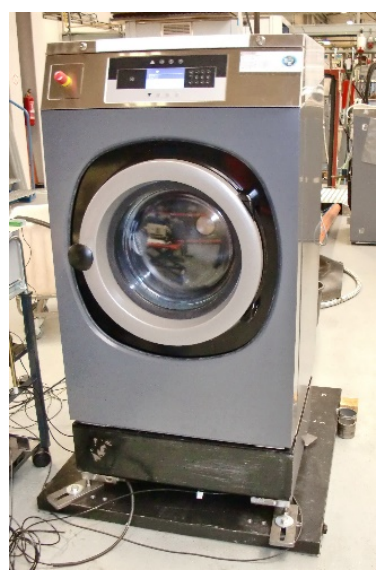

Figure 1. Typical industrial washing machine during measurement
Throughout the duration of the washing process the entire force cycle is evaluated with the maximum value. The aim of this paper is to quantify the properties of the maximal values.

The building structures are technically limited to a maximum load and a maximum floor deflection. So that knowledge of maximal forces is crucial for the correct positioning of the machines. Therefore, our industry partner must provide the maximal loading force in the technical parameters of the product. To solve the problem, we need to choose an appropriate distribution to describe the data. Another objective is to determine the minimum number of measurements that brings about quality results.

The problem of modelling and estimating the extreme value was presented in [Cole 2001, Folta 2012, Kracik 2013]. Generally, the usage of ordinary continuous distribution is sufficient for modelling extreme values [Cole 2001]. However, in some cases there is needed to apply a mixture distribution model [MCLachlan 2000]. In many cases there can be effectively used the mixture of normal distribution [McLahan 2000, Kracik 2013]. However, in modelling remote values the normal mixtures can be provided with many inaccuracies [Klemenc 2000]. Thus we present an alternative approach. We use a mixture of lognormal distributions instead of normal distributions. To estimate the parameters of the lognormal mixture we use the ExpectationMaximization (EM) algorithm [Dempster 1977, McLahlan 2000]. A modification of the EM algorithm will be briefly presented in this study.

\section{DETERMINING THE NUMBER OF DATA}

It is recommended to analyse at least $\mathbf{3 0}$ measurements in a standard data analysis. However, for determining higher quantile values it was necessary to use a larger data source [Cole 2001]. A simulation test was done to estimate the appropriate number of data. It randomly generated 3000 data sets (thousand per every distribution), each one with 1000 values with Weibull, Lognormal or Extreme-Value distribution. Thereafter there was randomly chosen from 40 to 100 measurements from every generated set. Finally, quantiles of generated sets and 40-100 values subsets were compared. It computed the relative frequency of the quantiles which did not overestimate the real value of the quantile. The results are presented in Table 1. According to Table 1 we can conclude that the minimal number of data is 100

\begin{tabular}{|l|l|l|l|l|}
\multirow{2}{*}{ Quantile } & \multicolumn{5}{|l}{ Amount of measurements } \\
\cline { 2 - 5 } & 40 & 60 & 80 & 100 \\
\hline 0.9 & 0.9 & 1 & 1 & 1 \\
\hline 0.91 & 0.90 & 1 & 1 & 1 \\
\hline 0.92 & 0.9 & 1 & 1 & 1 \\
\hline 0.93 & 0.89 & 1 & 1 & 1 \\
\hline 0.94 & 0.88 & 1 & 1 & 1 \\
\hline $\mathbf{0 . 9 5}$ & 0.88 & 0.99 & 1 & 1 \\
\hline $\mathbf{0 . 9 6}$ & 0.8 & 0.96 & 1 & 1 \\
\hline $\mathbf{0 . 9 7}$ & 0.79 & 0.98 & 1 & 1 \\
\hline $\mathbf{0 . 9 8}$ & 0.7 & 0.92 & 0.99 & 1 \\
\hline $\mathbf{0 . 9 9}$ & 0.67 & 0.91 & 3 & 1 \\
\hline $\mathbf{0 . 9 9 5}$ & 0.61 & 0.91 & 1 & 1 \\
\hline $\mathbf{0 . 9 9 9}$ & 0.52 & 0.90 & 0.94 & 0.99 \\
\hline
\end{tabular}

Table 1. Reliability of quantile estimate 


\section{PROCESS MEASUREMENT}

For correctly estimating the probable maximum it was need to conduct at least a hundred measurements per machine. Since repeated measurements of wash cycles, with the participation of technicians is ineffective, new software (presented in Figure 2) was developed to measure the process. According to experience from the previous measurements, the maximum values were measured only in the loosening and centrifugation mode because the dynamic effects of those modes are always significantly higher than during the washing process.

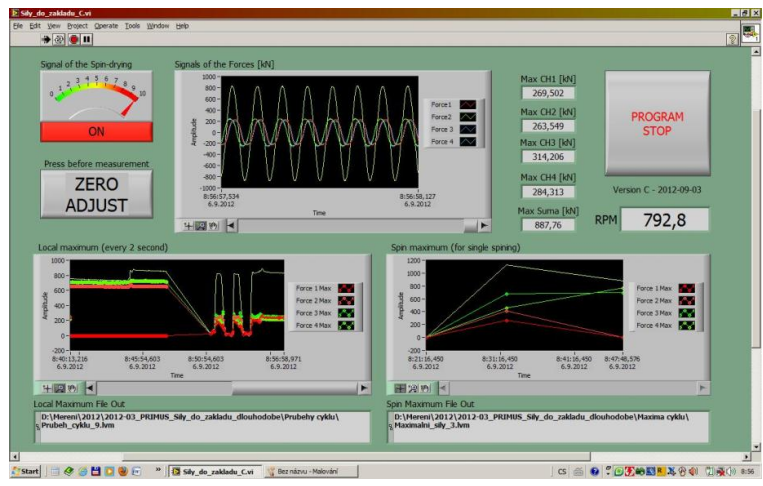

Figure 2. Front panel of the measuring software

The software was developed to measure the occurrence of peak values with the following characteristics:

- After obtaining the signal that the spinning and spinning mode is started, the software starts recording the force values acting on the floor from four different sensors located at the corners of the washing machine. The total vertical force was finally calculated as a sum of all four values.

- Software regularly evaluates the partial maximum value, and saves it in its memory. If the new value is larger than the previous value, it records this value as the maximum of the cycle.

- After finishing the spin cycle, the maximal values are saved 4 for each force sensor and one for the sum of the forces. Figures 4 and 6 present examples of the total maximum for washing machines per $36 \mathrm{~kg}$ of laundry and $70 \mathrm{~kg}$ of linen).

- Parallelly a set of local three-second maxima is created, which serves for the checking the remote values. It detects a possible electrical signal error (Figures 3 and 5).

- Finally, we obtain a database of maxims measured over the chosen time period, which can be statistically analysed in the form of histograms.

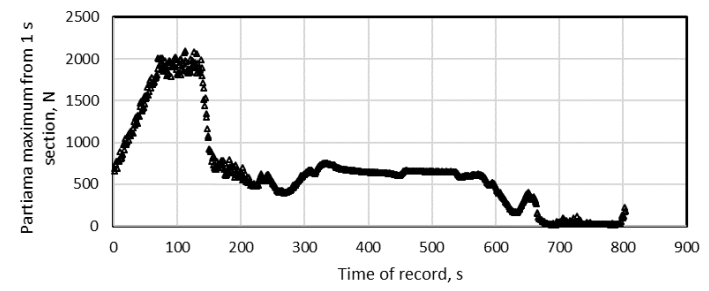

Figure 3. Record of partial maxima for one washing cycle - machine per $36 \mathrm{~kg}$

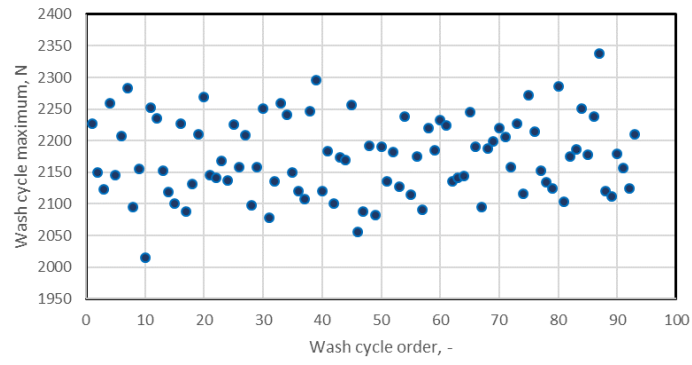

Figure 4. Maximums in separate washing cycles - machine per $36 \mathrm{~kg}$

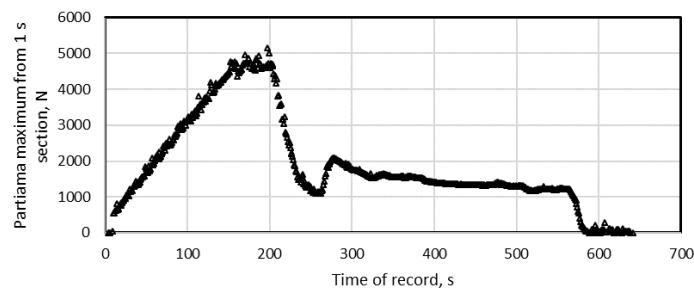

Figure 5. Record of partial maxima for one washing cycle - machine per $70 \mathrm{~kg}$

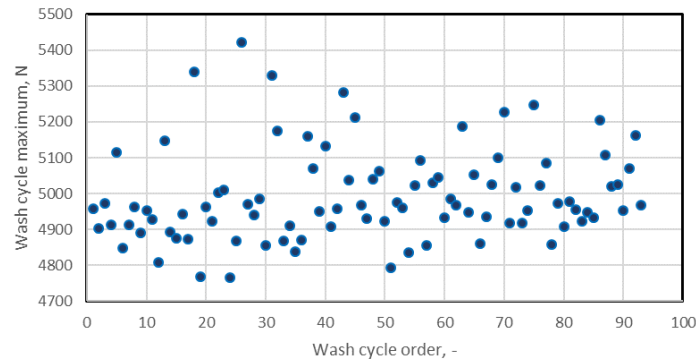

Figure 6. Maxima of separate washing cycles - machine per $70 \mathrm{~kg}$

\section{PROBLEM FORMULATION}

The aim of this paper is to present an analysis of industry washing machines. Nineteen different machines were analysed in the presented research. We focused on determining the probability of the higher values of operational forces, at the request of an industrial partner. Generally, the task corresponds to the search for higher quantile values. Since the number of measurements is about one hundred per machine, the data sources could not be analysed as the discrete variable. Therefore, the data files were approximated by various continuous probability distributions. The parameters of the used distributions were determined using a maximal likelihood approach. The distribution suitability was tested by the chisquare test and the Kolmogorov-Smirnov test. Three different distributions were used to describe the data sufficiently. In one case a convex mixture of lognormal distribution was used (see the table below). The parameters of the lognormal mixture were estimated using a modified Expectation-Maximization (EM) algorithm.

\begin{tabular}{|c|c|}
\hline Distribution & Number of applications \\
\hline Weibull & 8 \\
\hline Lognormal & 5 \\
\hline Extreme value & 5 \\
\hline Lognormal mixture & 1 \\
\hline
\end{tabular}

Table 2. Number of used distributions EM algorithm 
The EM algorithm is a method for finding the maximumlikelihood estimate of unknown parameters. The algorithm is an iterative method so that it converges with the local minimum of the likelihood function. The algorithm is applied to data with missing values (or it assumes the existence of missing data). The most used application of the algorithm is finding the parameters of mixture distribution. In this paper we have briefly presented a modification of the algorithm for finding the parameters of the mixture of lognormal distributions.

The algorithm consists of two steps E-stem (Expectation-step) and M-step (Maximization-step). It assumes the existence of observed data $X$ and unobserved data $Y$.

- E-step: In this step the algorithm computes the conditional expected value of the logarithmic loglikelihood function with respect to the parameter $\theta^{j}$ of the $j^{\text {th }}$ iteration and $X$.

$q_{J+1}(\Theta)=E_{\Theta}\left[\log L(\theta \mid X, Y) \mid X, \theta^{j}\right]$

- M-step: In this step the algorithm finds the $\theta^{j+1}$ that maximizes the expectation computed in the first step.

$\Theta_{j+1}=\operatorname{argmax} q_{j+1}(\Theta)$

Now we present the EM algorithm for estimating the parameters of mixture distributions. A mixture distribution can be described by the following formula

$$
f(x \mid \Theta)=\sum_{c=1}^{C} \alpha_{c} f\left(x \mid \theta_{c}\right)
$$

where

$\alpha_{1}, \alpha_{2}, \ldots, \alpha_{C}$ are nonnegative real numbers such that

$\sum_{c=1}^{C} \alpha_{c}=1$,

$f\left(x \mid \theta_{c}\right)$ is the distribution of $\mathrm{c}^{\text {th }}$ component

$\Theta=\left(\alpha_{1}, \alpha_{2}, \ldots, \alpha_{C}, \theta_{1}, \theta_{2}, \ldots, \theta_{C}\right)$ is the vector of estimated parameters of the mixture model.

After a series of technical adjustments, the E-step can be rewritten in the following form:

$$
\begin{aligned}
& q_{J+1}(\Theta) \\
& =\sum_{c=1}^{C} \sum_{i=1}^{N} \log \left(\alpha_{c}\right) f\left(c \mid x_{i}, \theta^{j}\right) \\
& +\sum_{c=1}^{C} \sum_{i=1}^{N} \log \left(f\left(x_{i} \mid \theta_{c}\right)\right) f\left(c \mid x_{i}, \theta^{j}\right)
\end{aligned}
$$

Using the former expression, we can maximize $\alpha_{c}, \theta_{c}$ from the form (1) independently.

Optimizing the first addition by applying the Lagrange multiplier we obtain an estimate of $\alpha_{c}$.

Substituting the density of the lognormal distribution with parameters $\mu_{c}, \sigma_{c}$ we rewrite the right part of the formula as:

$$
\begin{aligned}
& \sum_{c=1}^{C} \sum_{i=1}^{N} \log \left(f\left(x_{i} \mid \theta_{c}\right)\right) f\left(c \mid x_{i}, \theta^{j}\right) \\
& =\sum_{c=1}^{C} \sum_{i=1}^{N} \log \left(\frac{1}{x_{i} \sigma_{c} \sqrt{2 \pi}} e^{-\frac{\left(\ln x_{i}-\mu_{c}\right)^{2}}{2\left(\sigma_{c}\right)^{2}}}\right) f\left(c \mid x_{i}, \mu^{j}, \sigma^{j}\right)
\end{aligned}
$$

Taking the derivative with respect to $\mu_{c}, \sigma_{c}$, we obtain the maximal likelihood estimate of $\mu_{c}^{j+1}, \sigma_{c}^{j+1}$.

Generally, the maximal likelihood estimate of unknown parameters off lognormal mixture distribution of $j+1^{\text {th }}$ iteration takes the form:

$$
\alpha_{c}^{j+1}=\frac{1}{N} \sum_{i=1}^{N} f\left(c \mid x_{i}, \mu_{c}^{j}, \sigma_{c}^{j}\right)
$$

$$
\begin{aligned}
& \mu_{c}^{j+1}=\frac{\sum_{i=1}^{N} \log x_{i} f\left(c \mid x_{i}, \mu_{c}^{j}, \sigma_{c}^{j}\right)}{\sum_{i=1}^{N} f\left(c \mid x_{i}, \mu_{c}^{j}, \sigma_{c}^{j}\right)} \\
& \left(\sigma_{c}^{j+1}\right)^{2}=\frac{\sum_{i=1}^{N}\left(\log x_{i}-\mu_{c}^{j+1}\right) f\left(c \mid x_{i}, \mu_{c}^{j}, \sigma_{c}^{j}\right)}{\sum_{i=1}^{N} f\left(c \mid x_{i}, \mu_{c}^{j}, \sigma_{c}^{j}\right)}
\end{aligned}
$$

\section{RESULTS}

In this chapter we have presented four examples of analysed data. Every data source is described with a different distribution - Weibull, Lognormal, Extreme value and Gaussian mixture distribution. In the case of a widely used continuous distribution the appropriate distribution parameters were estimated using the maximum likelihood approach. The parameters of lognormal mixture distribution were estimated with the EM algorithm described above.

In next subchapters we will present the measured data, its histogram and the final evaluation of the probable maximum for different distributions. In Table 3 we describe summarized statistics of the presented data sets.

\begin{tabular}{|l|c|c|c|c|}
\hline \multicolumn{1}{|c|}{ Text } & Wbl. & Mix. Log & E.V. & Log. \\
\hline count & 100 & 110 & 95 & 150 \\
\hline mean & 980 & 2180 & 1050 & 1405 \\
\hline median & 1000 & 2155 & 960 & 1480 \\
\hline max & 1305 & 1305 & 1300 & 2155 \\
\hline Std. & 91 & 102 & 106 & 263.1 \\
\hline q. 0.99 & 1301 & 2340 & 1261 & 1920 \\
\hline q. 0.999 & 1320 & 2380 & 1342 & 2100 \\
\hline
\end{tabular}

Table 3. Summary statistics

\subsection{Weibull Distribution}

This distribution function was used to evaluate a washing machine with a load of $36 \mathrm{~kg}$ of laundry and for evaluating the centrifugation cycle only for a maximum speed. The graphical results are presented in Figures 7-9.

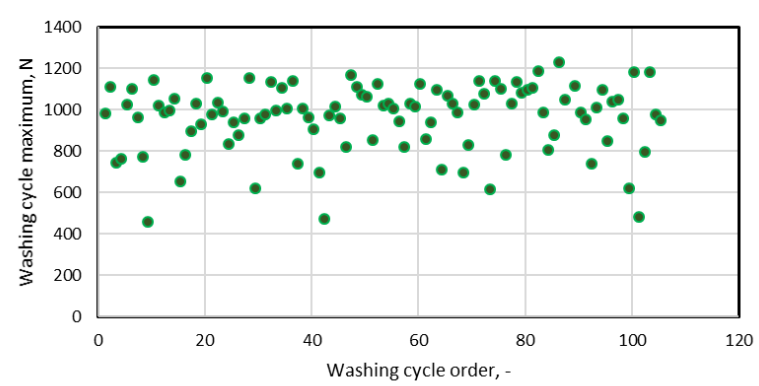

Figure 7. Maximums in separate washing cycles

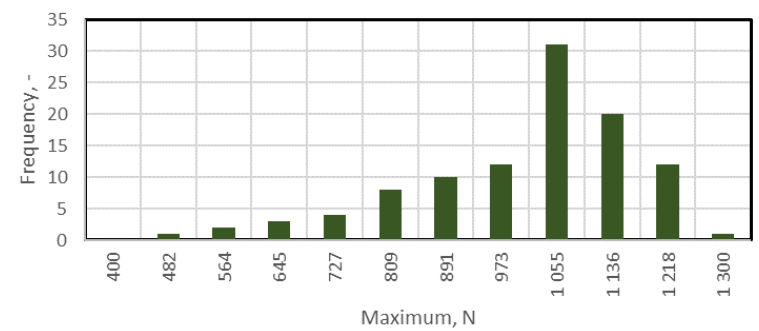

Figure 8. Histogram of maxima from Fig. 7 


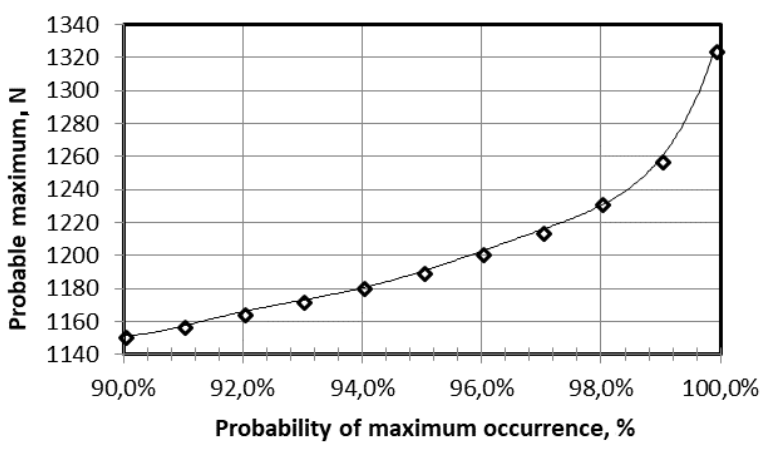

Figure 9. Estimation of the maximum force for different probability of occurrence for Weibull distribution

\subsection{Lognormal Mixture Distribution}

This distribution function was used to evaluate a washing machine with a load of $36 \mathrm{~kg}$ of laundry and for evaluating the entire centrifugation cycle. The graphical results are presented in Figures 10-12.

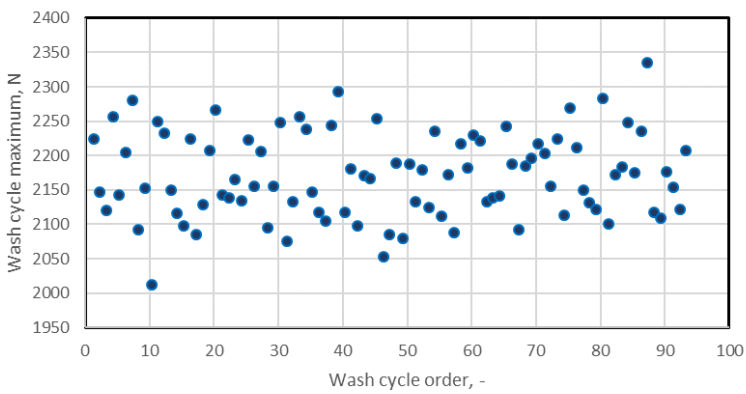

Figure 10. Maximums in separate washing cycles

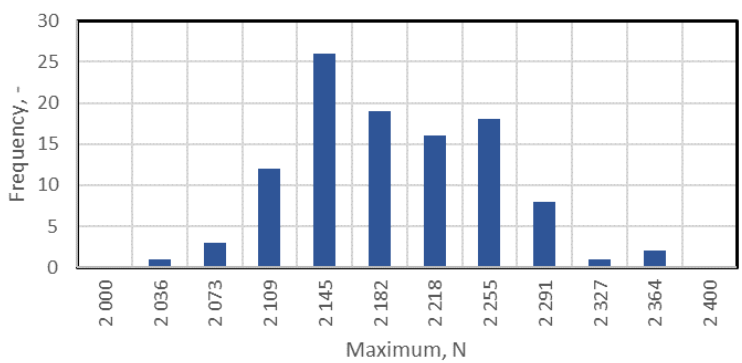

Figure 11. Histogram of maxima from Fig. 10

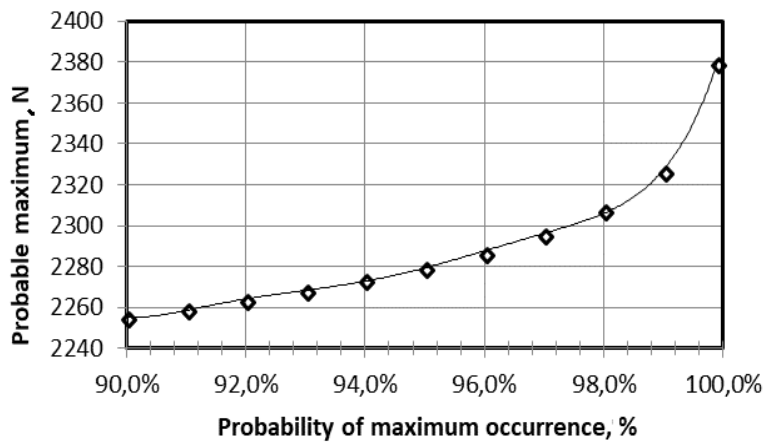

Figure 12. Estimation of the maximum force for different probability of occurrence for Lognormal Mixture distribution

\subsection{Extreme value}

This distribution function was used to evaluate a washing machine with a load of $50 \mathrm{~kg}$ of laundry and for evaluating the centrifugation cycle only for a maximum speed. The graphical results are presented in Figures 13-15.

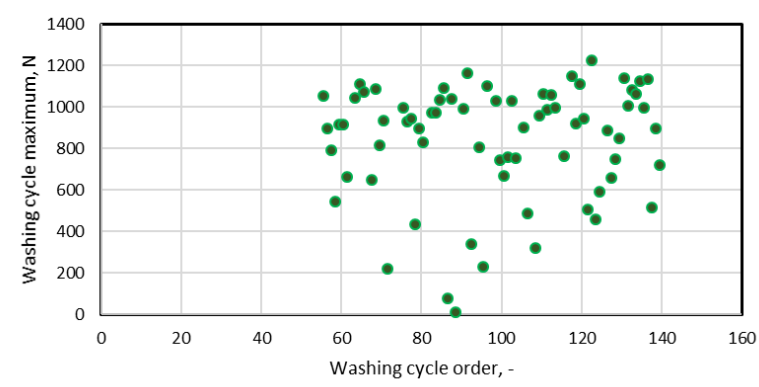

Figure 13. Maximums in separate washing cycles

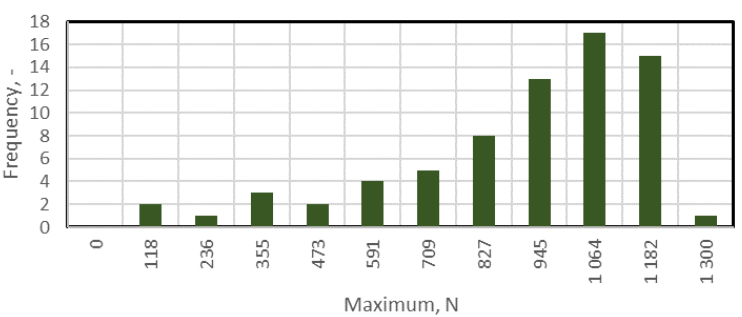

Figure 14. Histogram of maxima from Fig. 13

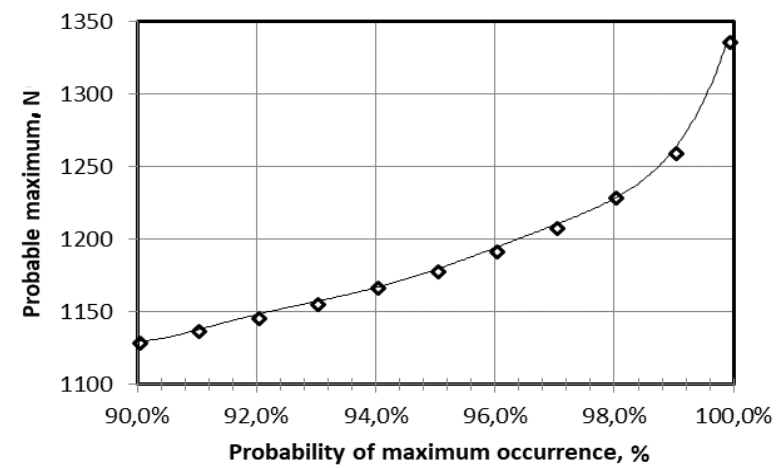

Figure 15. Estimation of the maximum force for different probability of occurrence for Extreme value distribution

\subsection{Lognormal distribution}

This distribution function was used to evaluate a washing machine with a load of $70 \mathrm{~kg}$ of laundry and for evaluating the entire centrifugation cycle. The graphical results are presented in Figures 16-18.

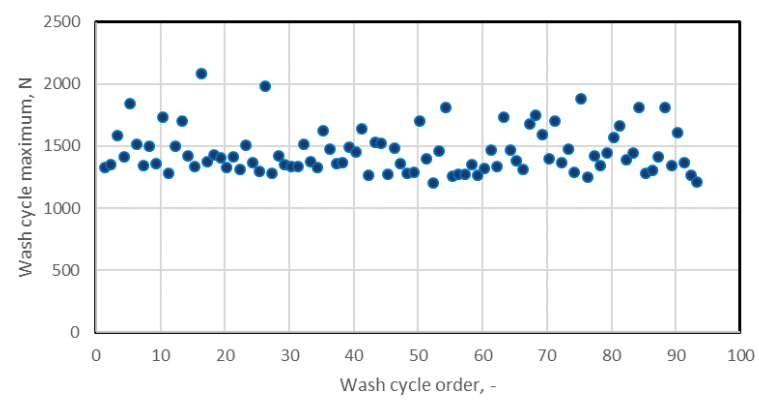

Figure 16. Maximums in separate washing cycles 


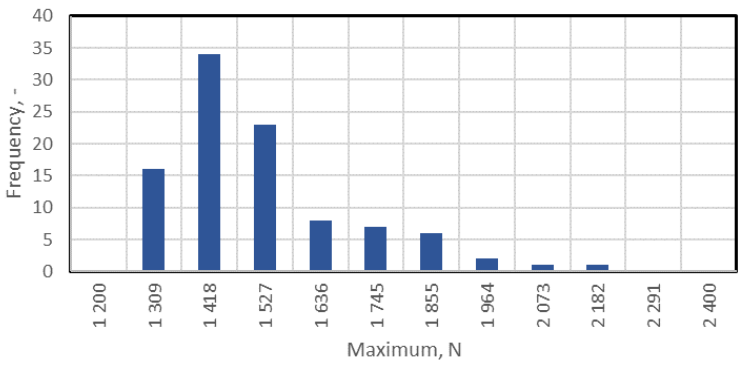

Figure 17. Histogram of maxima from Fig. 16

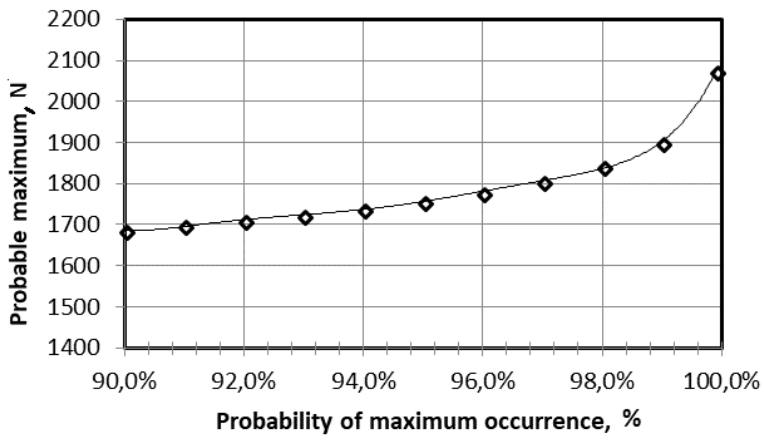

Figure 18. Estimation of the maximum force for different probabilities of occurrence for Lognormal distribution

\section{CONCLUSION}

A statistical analysis of the maximal forces was presented in this study. Analyzed data were described using various continuous distributions - Weibull, Extreme value and Lognormal distribution. The last row of Table 3 shows the resulting values of the probable maximum value for $99.9 \%$. These data suggest the need for a proper analysis of the type of probability distribution, because using the wrong distribution can lead to incorrect results.

In one case it was necessary to use a convex combination of lognormal distributions. The parameters were estimated using a modified EM algorithm. Since we are describing the extreme values, the lognormal mixture modelling is further more effective than the more often used normal mixture distribution. This technique can be applied in a wide area of statistical analysis and applications.

\section{REFERENCES}

\section{Book:}

[McLahlan 2000] McLachlan, G., Peel, D. Finite Mixture Models. New York: John Wiley \& Sons, 2000. ISBN: 9780471006268.

\section{Paper in a journal:}

[Dempster 1977] Dempster, D., Laird, N. M., Rubin, D. B. EM algorithm Maximum Likelihood from Incomplete Data via the EM Algorithm. Journal of the Royal Statistical Society, April 1977, Vol. 39, No. 1, pp.1-37. ISSN: 1467-9868.

[Klemenc 2000] Klemenc, J., Fajdiga, M. Description of statistical dependencies of parameters of random load states. International journal of fatigue, May 2000, Vol 22, No.5, pp 357-367. ISSN: 0142-1123.

\section{Paper in proceedings:}

[Coles 2001] Coles, S. An Introduction to Statistical Modeling of Extreme Values. Springer, 2001. ISBN 978-1-4471-3675-0.

[Folta 2012] Folta, Z., Skalny, P. Determination of probable maximum of the stochastic signal. In Proceedings 53rd International Conference of Machine Design Departments, Brno, VUT Brno, 2012.

[Kracik 2013] Kracik, J., Skalny P. Analysis of Time Evolution of Load Cycles in Steel Structures. Safety, reliability and risk analysis: beyond the horizon, CRC Press, September, 2013, pp. 1419-1425, ISBN 978-1-138-00123-7.

\section{CONTACTS:}

Doc. Ing. Zdenek Folta, Ph.D.

VSB-Technical University of Ostrava, Ostrava, Czech Republic / Department of Machine Parts and Mechanisms 17. listopadu 2172/15, Ostrava-Poruba, 708 00, Czech Republic +420 596993 393, zdenek.folta@vsb.cz, www.vsb.cz

Mgr. Pavel Skalny, Ph.D.

VSB-Technical University of Ostrava, Ostrava, Czech Republic / Department of Machine Parts and Mechanisms 17. listopadu 2172/15, Ostrava-Poruba, 708 00, Czech Republic +420 596994 152, pavel.skalny@vsb.cz,www.vsb.cz

Ing. Petr Matejka

Alliance Laundry CE s.r.o.

Mistecka 1116, Pribor, 742 58, Czech Republic

+420 556768 800, petr.matejka@alliancels.cz, primuslaundry.com

Ing. Miroslav Trochta, Ph.D.

VSB-Technical University of Ostrava, Ostrava, Czech Republic / Department of Machine Parts and Mechanisms 17. listopadu 2172/15, Ostrava-Poruba, 708 00, Czech Republic

+420 596993 285, miroslav.trochta@vsb.cz, www.vsb.cz

Ing. Daniel Pistacek, Ph.D.

VSB-Technical University of Ostrava, Ostrava, Czech Republic / Department of Machine Parts and Mechanisms 17. listopadu 2172/15, Ostrava-Poruba, 708 00, Czech Republic

+420 596993 235, daniel.pistacek@vsb.cz,www.vsb.cz 